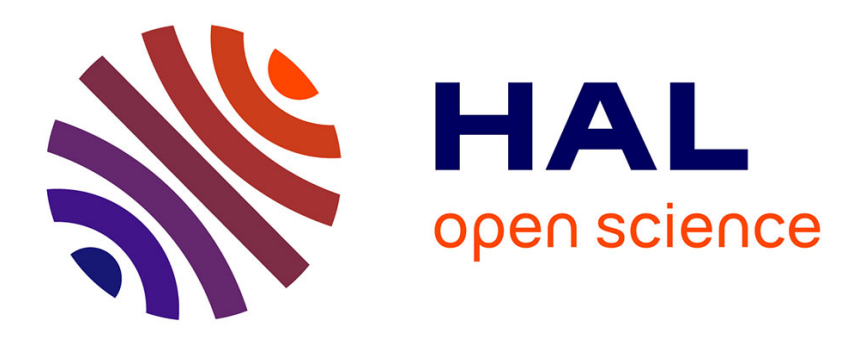

\title{
Autour des legiones Cannenses (2)
}

Sandra Péré-Noguès

\section{To cite this version:}

Sandra Péré-Noguès. Autour des legiones Cannenses (2). Pallas. Revue d'études antiques, 1998, 48, pp.225-232. halshs-00781616

\section{HAL Id: halshs-00781616 https://shs.hal.science/halshs-00781616}

Submitted on 28 Jan 2013

HAL is a multi-disciplinary open access archive for the deposit and dissemination of scientific research documents, whether they are published or not. The documents may come from teaching and research institutions in France or abroad, or from public or private research centers.
L'archive ouverte pluridisciplinaire HAL, est destinée au dépôt et à la diffusion de documents scientifiques de niveau recherche, publiés ou non, émanant des établissements d'enseignement et de recherche français ou étrangers, des laboratoires publics ou privés. 


\section{Autour des legiones Cannenses (2)}

Sandra PÉRÉ-NOGUÈS (U.T.A.H., Université de Toulouse-Le Mirail)

La déroute des troupes romaines à Cannes en 216 avant J.-C. avait conduit le Sénat à prendre de sévères mesures disciplinaires à l'encontre des rescapés de la bataille, les legione Cannenses. Le récit de Tite-Live offre un témoignage intéressant sur leur destin durant la seconde guerre punique: d'abord reléguées en Sicile où ces légions ont sans doute participé à quelques opérations grâce au soutien politique de Marcellus, les troupes vaincues à Cannes ont pu laver leur déshonneur en intégrant les rangs de l'expédition africaine conduite par Scipion en 204 avant notre ère.

Si l'épisode des legiones Cannenses reste surtout connu par le récit de Tite-Live, il n'a pas échappé à deux auteurs latins, Valère Maxime et Frontin. Leurs projets littéraires répondent évidemment à des objectifs fort différents de ceux de l'œuvre livienne, mais leurs références aux légions de Cannes permettent de comprendre comment le souvenir d'un épisode aussi dramatique de l'histoire de Rome a pu se perpétuer.

Dans un précédent article sur les legiones Cannenses à travers l'œuvre de Tite-Livel, des zones d'ombre étaient apparues sur les heures qui avaient immédiatement suivi la victoire des Carthaginois. D'une part, un complot mené par Caecilius Métellus et destiné présence de Scipion, alors tribun militaire romaine. D'autre part, Tite-Live signalait la projet de ses compagnons. Ces deux épirodes qui neves s'opposer de façon véhémente au des legiones Cannenses sont tradition déjà bienses ét sont egalement repris par Valère Maxime et Frontin, mais selon la tradition déjà bien établie par l'historien padouan.

Dans son ouvrage, Faits et Dits Mémorables, publié vers $31 / 32$ de notre ère, et composé à partir des témoignages de Cicéron, Salluste, Denys d'Halicarnasse et surtout Tite-Live, Valère Maxime reprend dans ses exempla l'histoire des légions de Cannes. $S^{\prime}$ il

S. Péré-Noguès, 1997, p. 121-130.

Sur sa volonté de composer un recueil d'exempla : Valère Maxime, Tome I, Introduction, p. 19-20 (CUF). 
n'apporte aucun élément nouveau au récit, il rappelle l'anecdote sur un ton tout à fait particulier :

"C'est la même colère que le Sénat a brandie contre ceux qui avaient abandonné la patrie à Cannes. Car la gravité de sa décision les avait placés au-delà de la condition des morts et, quand il reçut de Marcellus une lettre par laquelle celui-ci demandait le droit d'avoir recours à leur service pour le siège de Syracuse, il lui répondit qu'ils ne méritaient pas d'être reçus dans un camp, mais qu'il l'autorisait à faire ce qu'il jugerait conforme à l'intérêt de l'État, pourvu qu'aucun d'entre eux ne fût exempté de corvées, ne reçût de récompense militaire ni ne revînt en Italie tant que l'ennemi s'y trouvait. Voilà comment, quand on manque de vigueur, se manifeste la haine qu'on inspire à la valeur. „”

Cette référence aux legiones Cannenses est insérée dans une énumération d'exempla concernant la discipline militaire. Elle suit ainsi un autre exemple de punition militaire infligée par le Sénat aux prisonniers romains de Pyrrhus : à l'époque, les soldats furent dégradés et obligés de se montrer deux fois victorieux contre le roi d'Epire ${ }^{4}$. Mais dans le cas des anciens de Cannes, la décision est plus grave : il faut bien comprendre que le Sénat ne leur a pas accordé de seconde chance. Cela expliquerait la formule de Valère Maxime selon laquelle les soldats furent placés " ultra mortuorum condicionem ".

Dans le quatrième livre de ses Stratagemata 5 , ouvrage publié dans les années 85/95 de notre ère, Frontin a laissé lui aussi quelques notes intéressantes sur les legiones Cannenses

"Lors du consulat de Quintus Fulvius et Appius Claudius, les soldats, qui après la bataille de Cannes avaient été bannis en Sicile par le Sénat, supplièrent le consul Marcellus d'être menés au combat. Marcellus consulta le Sénat, qui déclara que ce n'était pas sa

3 Valère Maxime 2,7,15c: " Parem iram senatus adversus illos dextrinxit qui apud Cannas rem publicam deseruerant. Nam cum eos grauitate decreti ultra mortuorum condicionem relegasset. acceptis a M. Marcello litteris ut eorum sibi opera ad expugnationem Syracusarum uti liceret, rescripsit indignos esse qui in castra reciperentur, ceterum se ei permittere ut faceret quod expedire rei publicae iudicasset, dum ne quis ex his munere uacaret aut dono militiae donaretur aut in Italiam donec hostes in ea essent accederet. Sic enruis animos odisse uirtus solet. " (traduction R. Combès, CUF).

Valère Maxime, 2,7,15b : "Ils retrouvaient en effet dans leur mémoire combien avait été vigoureuse la sévérité que leurs ancêtres avaient manifestée pendant la guerre contre Tarente. Les forces de la nation y avaient été ébranlées et brisées, mais quand ils reçurent à Rome un grand nombre de leurs concitoyens, qui avaient été faits prisonniers et que le roi Pyrrhus avai renvoyés de sa propre initiative, ils décidèrent que ceux d'entre eux qui avaient servi comme cavaliers feraient leur service militaire dans l'infanterie, que ceux qui avaient été fantassins passeraient dans les groupes auxiliaires de frondeurs, qu'aucun parmi eux ne s'installerait dans le camp, n'entourerait la position qui leur avait été assignée en dehors de celui-ci d'une levée de terre ou d'un fossé, ne posséderait de tente faite de peau de bête. Quant à leur retour à leur position précédente dans l'armée, ils le proposèrent à tous ceux qui auraient ramené deux fois des dépouilles de l'ennemi. Ces châtiments contraignirent des hommes qui n'avaient été que l'aumône honteuse de Pyrrhus à devenir ses ennemis les plus acharnés."

5 Ce livre IV n'aurait pas été écrit par Frontin. Sur ce point et les sources utilisées par cet auteur (en particulier sa dépendance par rapport à Valère Maxime) : Frontin, Introduction, p. XXXXII (Loeb). volonté que le bien public fût confié à ceux qui s'étaient montrés déloyaux. Néanmoins il autorisa Marcellus à faire ce qui lui semblerait le mieux, à condition qu'aucun des soldats ne fût exempté de corvées, ne reçût de cadeau ou de récompense, ou ne revînt en Italie, tant qu'il y avait des Carthaginois dans le pays. "6

Tout comme pour Valère Maxime, c'est le récit de Tite-Live qui a inspiré Frontin Mais le ton sur lequel est relaté l'épisode s'écarte du caractère moralisateur très présen dans l'œuvre de Valère Maxime. Outre cet exemple qui s'inscrit dansè présen déve développement que Frontin consacre à la discipline militaire, il ajoute un autre type de mesures prises pendant le conflit : "Les légions qui avaient refusé de servir dans la guerre punique furent envoyées dans une sorte de bannissement en Sicile, et par vote du Sénat furent mises aux rations d'orge pendant sept ans $»^{7}$. Ce fait pourrait confirmer le récit de Tite-Live sur la politique disciplinaire sans concession menée par le Sénat à l'égard de tous les soldats et citoyens qui ne remplissaient pas leur devoir. En tors à une remplissaient pas alors à une remise en ordre des affaires militaires. A la fin de l'année 216, c'est-à-dire au lendemain des élections des magistrats ${ }^{8}$, il décide du sort des legiones Cannenses, à savoi leur relégation en Sicile jusqu'à la fin des hostilités9. Puis, vers le mois de mars 215, lor de la séance inaugurale, la décision est prise de leur confisquer la solde. Ces premières dispositions concernent l'ensemble des rescapés de Cannes, fantassins et cavaliers.

Le Sénat prend donc une mesure immédiate, destinée sans doute à frapper les esprits par sa sévérité. Mais cette volonté de faire un exemple ne se limite pas à ces seules sanctions : en 211, en séance inaugurale, interdiction leur est faite de s'installer près de villes ${ }^{10}$; en 209 , les cavaliers sont chassés des centuries équestres par décision des censeurs ${ }^{11}$ et obligés de prolonger leur service. De par ces mesures, il apparaît clairement que le châtiment des légions de Cannes, qui s'aggrave au cours de cette période, revêt un caractère exceptionnel : d'abord pénalisés sur le plan militaire, les soldats, en particulier les cavaliers, subissent donc une punition civique. Comme le remarque $\mathrm{Cl}$. Nicolet ${ }^{12}$, ces sanctions ont très tôt posé un problème politique : dès 212 , une députation formée par l'élite des légions de Cannes se rend auprès de Marcellus en Sicile pour exposer leurs récriminations ${ }^{13}$

6 Frontin, IV,1,44: " Q. Fulvio Appio Claudio consulibus milites ex pugna Cannensi in Siciliam a senatu relegati postulauerunt a consule $M$. Marcello, ut in proelium ducerentur. Ille senatum consuluit ; senatus negauit sibi placere committi his rem publicam, qui eam deseruissent; Marcello tamen permisit facere, quod videretur, dum ne quis eorum munere uacaret neue donaretur neue quod praemium ferret aut in Italiam reportaretur, dum Poeni in ea fuissent. " (traduction de l'auteur).

7 Frontin, IV,1,25: "Legionibus, quae Punico bello militiam detractauerant, in Siciliam uelut relegatis per septem annos hordeum ex senatus consulto datum est. ".

Nous avons suivi la chronologie établie par M. Bonnefond-Coudry, 1989, p. 263-267.

Tite-Live XXIII,25,7 (traduction A. Flobert, GF).

10 Tite-Live XXVI,1,10.

1 Tite-Live XXVII,11,14

2 Cl. Nicolet, 1976, p. 153.

S. Péré-Noguès, 1997, p. 124-125 
En outre, la punition infligée aux legiones Cannenses s'étend à d'autres catégories de soldats durant le conflit. En 215, dans la foulée de la décision prise contre ceux de Cannes, sont aussi relégués «les moins bons éléments de l'armée du dictateur », mais pour la durée légale de leur service. L'année suivante, Tite-Live précise que "les censeurs se consacrèrent au redressement moral et au châtiment des fautes que la guerre avai occasionnées, comme ces affections qui se déclarent à la suite d'une longue maladie et qui sont sécrétées par l'organisme quand il est atteint "14. Parmi les diverses mesures prises, " [les censeurs] tirèrent de la liste des mobilisables le nom de ceux qui n'avaient pas fait la guerre ces quatre dernières années et ne pouvaient se justifier par une dispense valable ou un congé de maladie : il y en avait plus de deux mille, qui furent déchus de leurs droits et dégradés. Les sénateurs aggravèrent la sentence si rigoureuse des censeurs par cette sévère décision : tous ceux qui auraient reçu un blâme des censeurs serviraient dans l'infanterie ; on les expédierait en Sicile, où ils retrouveraient les débris de l'armée de Cannes qui ne devait pas être libérée avant que l'ennemi ait été chassé d'Italie »15.

Enfin, en 211, la même mesure est encore appliquée aux rescapés de l'armée de $\mathrm{Cn}$. Fulvius battue à Herdonea ${ }^{16}$. La récurrence de ces sanctions montre d'abord la détermination du Sénat, soutenu par les censeurs, à réprimer tous les manquements des soldats au salut de la cité. Pour parvenir à cet objectif, le Sénat se dote d'un instrument de répression, une sorte de châtiment collectif, qui s'applique autant aux citoyens récalcitrants qu'aux troupes vaincues. Dans le cas des légions de Cannes, et vraisemblablement dans celui des troupes d'Herdonea, le châtiment se veut exemplaire, voire exacerbé, de manière à " motiver " le moral des troupes et surtout à prévenir toute volonté d'insoumission, voire d'opposition, à la politique de la cité.

Dans son exemple, Frontin reprend donc une tradition déjà présente chez Tite-Live : il est difficile d'en contester la véracité dans la mesure où Tite-Live s'appuie vraisemblablement sur des documents officiels, de nature annalistique, concernant l'activité sénatoriale.

Frontin enrichit aussi son récit d'un détail plus technique : la substitution de l'orge aux rations de blé appartient effectivement à l'éventail des punitions infligées aux mauvais soldats ${ }^{17}$, pratiques que devait bien connaître l'ancien chef militaire qu'il fut. Son témoignage reste aussi très important car il souligne le rôle de la Sicile comme lieu de bannissement pendant le conflit. Cette idée déjà perceptible chez Tite-Live semble assez paradoxale car jusque dans les années 212/211, la Sicile a constitué un enjeu important dans les stratégies de Rome et Carthage ${ }^{18}$.

A l'instar de Tite-Live, Valère Maxime et Frontin ont bien ressenti la gravité de la sanction prise par le Sénat à l'encontre des vaincus de Cannes. Ils fournissent évidemment une raison très morale pour justifier la réaction sénatoriale : les legiones Cannenses n'ont-

4 Tite-Live XXIV,18,2.

15 Tite-Live, XXIV,18,7-9.

16 Tite-Live XXVII,7,12-13.

17 Plutarque, Marcellus, 25; Suétone, Auguste, 24.

18 Voir P. Marchetti, 1972 , p. 5-26. elles pas voulu abandonner la patrie ? Ainsi, comme dans le récit de Tite-Live, la réaction sénatoriale ne saurait se comprendre sans faire allusion au complot de Métellus.

Rappelons brièvement les faits. Après le rassemblement des débris de l'armée romaine à Canusium, les cadres de l'état major essaient de prendre des dispositions sur la conduite des futures opérations. Tite-Live décrit ainsi la situation:

"Parmi les réfugiés se trouvaient des tribuns militaires:

la première légion, fils du dictateur de l'année précédes: Quintus Fabius Maximus, de Publius Cornélius Scipion précédente; Lucius Publius Bibulus e troisième légion, t'óne légion, qui venait d'exercer : tous furent d'accord pour partager le commandement suprême entre Publius Scipion, qui était encore très jeune, et Appius Claudius. Comme ils discutaient entre eux de la situation générale, Publius Furius Philus, le fils de l'ancien consul, leur déclara qu'il était bien illusoire de nourrir de vaines espérances : la République était perdue, la République était morte; de jeunes nobles, dirigés par Marcus Caecilius Métellus, songeaient à prendre la mer et à s'embarquer, avec l'intention d'abandonner l'Italie et de se réfugier à la cour de quelque roi.

Ce crime, affreux par lui-même, qui venait s'ajouter inopinément à tant de drames, laissa l'assistance sans réaction, sous le coup de la surprise et de l'étonnement : on demandait láunio et de l'étonnement : on destins avaient conseil de guerre discussions discussions; que ceux qui veulent sauver la patrie prennent leurs armes et me suivent! l'endroit où on trame de tels complots est à strictement parler un camp ennemi. "

Accompagné de quelques hommes il se rendit directement au logis de Métellus et $\mathrm{y}$ trouva rassemblés les jeunes gens dont on lui avait parlé ; maintenant son épée au-dessus de la tête des conspirateurs, il prononça ces paroles : "Je jure en mon âme et conscience de ne pas abandonner la République romaine et Je jure en mon âme et conscience l'aban pas tolérer qu'un citoyen l'abandonne. Si je manque sciemment à mon serment, que Jupiter Très Bon Très Grand frappe ma personne, ma maison, ma famille, mes biens, des malheurs les plus affreux. J'exige, Marcus Caecilius que tu répètes ce serment, et vous tous à sa suite. Si quelqu'un refuse de jurer, qu'il le sache, je le passerai au fil de mon épée. " Aussi épouvantés que s'ils se trouvaient en présence d'Hannibal, le général victorieux, tous prêtèrent serment et se mirent à la disposition de Scipion. "19

Cet épisode a suscité de multiples interrogations chez les historiens modernes. Faut-il tout d'abord accorder un crédit quelconque au fameux complot de Métellus ? Il serait difficile de mettre en doute l'existence de ce complot qui est attesté par de nombreux témoignages littéraires s'inspirant directement ou non de Tite-Live. En effet, Valère Maxime suit dans son récit la tradition livienne :

"Il n'a pas péri de ce qu'il faisait pour l'État, le premier Africain, mais sa valeur admirable lui a permis d'éviter que l'État ne pérît, puisque, au moment où la défaite de Cannes avait abattu notre ville et fait qu'elle semblait ne plus être que la proie de son vainqueur, qui était Hannibal, au moment où cette situation poussait ceux qui

19 Tite-Live, XXII, 53. 
survivaient, après l'écrasement de notre armée, à projeter de s'enfuir hors de l'Italie, à l'appel de Quintus Métellus, lui, qui n'était que tribun militaire, et tout jeune encore, il sortit son épée et, en les menaçant de mort l'un après l'autre, il les obligea tous à jurer qu'ils n'abandonneraient jamais leur patrie : le respect qu'il ressentait pour ses obligations, non seulement il l'a manifesté lui-même dans toute sa profondeur, mais, quand [ce respect] abandonnait le cœur des autres, il l'y a fait revenir. »20

Dans un style plus dépouillé, Frontin décrit ainsi l'épisode :

"Après le désastre de Cannes, comme les Romains étaient si terrorisés qu'une large partie des survivants pensèrent abandonner l'Italie, avec l'accord de nobles de très haut rang, Publius Scipion, alors extrêmement jeune, lors de la même assemblée où une telle affaire était discutée, proclama avec une grande véhémence qu'il tuerait de ses propres mains quiconque refuserait de passer un serment selon lequel il ne nourrissait pas de projet d'abandonner l'État. S'étant d'abord lié par un tel serment, il tira son épée et menaça de mort l'un de ceux qui se tenaient tout près de lui, alors qu'il aurait aussi passé serment. Cet homme fut contraint par crainte de jurer allégeance : le reste fut obligé par l'exemple du premier $» .21$

Deux écrivains latins ont eux aussi fait référence au complot de Métellus et perpétué le souvenir de l'action héroïque de Scipion. Il s'agit d'abord du poète padouan du premier siècle de notre ère, Silius Italicus, qui donne au discours de Scipion un aspect plus apologétique :

"Père qui as fait du temple tarpéien ta seconde demeure après le ciel et toi, Saturninienne, que les maux d'Ilion n'ont point encore changée et toi, vierge redoutable, ceinte des fureurs de la Gorgone grâce à l'égide qui protège ta poitrine et vous, dieux indigètes, de par ma volonté unie à vos divines injonctions, par la tête d'un père magnanime, à nulle autre divinité inférieure pour moi, je jure : jamais je n'abandonnerai le royaume de Lavinius, ni ne souffrirai qu'on l'abandonne, tant que la vie me restera. Sur-le-champ, va, prends les dieux à témoin que si les torches libyennes embrasent un jour les remparts, tu n'auras pas l'audace, Métellus, de porter tes pas sur aucune autre terre. Si tu ne prononces un tel serment, celui qui te fait trembler, qui interrompt par la terreur ton sommeil, Hannibal, est là en armes devant toi : tu mourras, aucun Punique ne m'aura de sa mort procuré une gloire de meilleur aloi ". Le choc de ces menaces fit tomber le projet, et déjà, suivant l'ordre prescrit, ils enchaînent leur vie à celle de la patrie et prononcent, face aux dieux, les serments dictés et purifient leurs cœurs criminels. „22

Même au Vème siècle de notre ère, un historien chrétien aussi polémiste qu'Orose fait allusion à ce singulier épisode :

"Ce qui restait de Romains perdit si complètement l'espoir dans la République que les sénateurs pensèrent prendre la décision d'abandonner l'Italie et de se mettre en quête d'un lieu pour s'installer. Ce qui aurait été ratifié, sur la proposition de Caecilius Métellus, si Cornélius Scipion, alors tribun militaire, le même qui sera plus tard "l'Africain ", ne

20 Valère Maxime, 5,6,7

21 Frontin, IV,7,39.

22 Silius Italicus, La Guerre Punique, X,432-447. l'avait empêché en brandissant son glaive, et n'avait contraint les sénateurs à prêter plutôt avec lui le serment de défendre la patrie. ${ }^{23}$

Notons d'abord que ces récits sont unanimes quant au responsable du complot, Métellus. Orose livre une version plus surprenante de l'épisode: c'est le Sénat à l'instigation de Métellus qui est prêt à abandonner l'Italie. En revanche le lieu choisi pou l'exil reste obscur ; certains historiens modernes ont voulu y reconnaître la Macédoine.

Toutefois, même si ces témoignages sont tardifs et vraisemblablement éloignés de la réalité, le complot reste fort probable, compte tenu du désarroi qui s'est abattu sur les rangs de l'armée romaine après sa déroute. Ajoutons que Tite-Live rend compte dans son récit de la carrière politique de Métellus. Ce n'est qu'en 209 avant notre ère que ce aristocrate est effectivement puni à l'ex. 209 avant notre ère que ce rayé de la liste des sénateurs au connes : il est alor l'Italie après le désastre de Cars motif qu'il s'était "déshonoré en proposant de quitte

Si la tentative de "trahison " de Métellus paraît plausible sans qu'il soit possible d'en cerner le déroulement, la présence de Scipion au moment du désastre de Cannes cause un certain embarras 25 . En effet, c'est en tant que tribun militaire qu'il a participé à la bataille Or, Tite-Live laisse planer un doute sur les responsabilités des soldats et de l'état major dans cette défaite, lorsqu'il rapporte le discours de Scipion à ses soldats à la veille de minimiser le rôle de Scipion dans l'échec des a trouvé ainsi un moyen judicieux de minimiser le rôle de Scipion dans l'échec des troupes romaines à Cannes. Cette volonté s'inscrit dans la construction de la geste de Scipion, futur grand vainqueur des - Tite-Live a réussi à faire un acte de bravoure destiné à servir d'exemplum aux futurs généraux. En outre cette idée est corroborée par le fait que l'épisode hérö̈que qui met Scipion aux prises avec les traîtres reste trop fortement suspect pour être vraiment crédible.

Les écrivains latins, qui ont pour la plupart puisé dans la source livienne, ont suivi, voire amplifié, cette tradition qui s'inscrivait parfaitement dans la mentalité romaine. Que l'on songe au sacrifice exemplaire du consul M. Atilius Régulus lors de la première guerre punique, symbole du courage et de la vertu romaine.

Ainsi l'épisode des legiones Cannenses dépasse le cadre strict de l'histoire militaire romaine durant la seconde guerre punique. A partir d'un châtiment collectif qui relevait

23 Orose, Histoires (contre les Païens), IV, 16, 6.

24 Tite-Live, XXVII,11,12. Voir aussi S. Péré-Noguès, 1997, p. 126-127.

25 Sur le problème de la présence de Scipion à Cannes: 12 . 126-127.

Ridley montre très justement de Scipion à Cannes : R.T. Ridley, 1975, p. 161-165. R.T. Scullard, a eu des difficultés pour comment un grand spécialiste de Scipion, comme H.H. conduit a de polye l'avait d'abord conduit à refuser toute véracité à cette épisode ; mais l'état fragmentaire du récit polybien ne permettant pas d'assurer une telle hypothèse, compte tenu de l'existence d'une monnaie de Canusium qui pourrait représenter Scipion, il est finalement parvenu à la conclusion que le tribun était présent à Cannes.

Tite-Live, XXIX,24,11-13 : “... Scipion ne méprisait nullement cette catégorie de soldats, lui qui savait [...] que la défaite de Cannes n'était pas due à leur lâcheté .... " (traduction P.
François, CUF). 
des règles en usage dans le domaine de la disciplina ${ }^{27}$, le Sénat a voulu faire un exemple et montrer sa sévérité et son pouvoir à l'égard de tout citoyen qui ne se conformait pas à la défense de la cité. De fait, il réussit à punir, même après quelque retard, un aristocrate tel que Caecilius Métellus. Son destin, ainsi que la carrière de Scipion, restent donc profondément liés au sort des anciens de Cannes.

Tite-Live a réussi à construire sur ce point un récit cohérent et orienté vers la mise en valeur du caractère d'une personnalité centrale de ce conflit, Scipion. A sa suite, les auteurs latins ont repris cette tradition concernant une période sombre de l'histoire de Rome. Mais ce lien originel entre les legiones Cannenses, Métellus et Scipion a peu à peu disparu pour laisser place à la geste héroïque du vainqueur d'Hannibal.

\section{Bibliographie}

Bonnefond-Coudry M., 1989, Le Sénat de la République. De la guerre d'Hannibal à Auguste : pratiques délibératives et prise de décision (BEFAR, 273), Rome.

Marchetti P., 1972, La deuxième guerre punique en Sicile. Les années 215-214 et le récit de Tite-Live, Bulletin de l'Institut Historique belge de Rome, 42, p. 5-26.

Nicolet Cl., 1976, Le métier de citoyen dans la Rome républicaine, Paris.

Péré-Noguès S., 1997, Note sur les legiones Cannenses: soldats oubliés de la deuxième guerre punique ?, Mél. C. Domergue, Pallas, 46, p. 121-130.

Ridley R.T., 1975, Was Scipio Africanus at Cannae ?, Latomus, 34, p. 161-165.

Scullard H.H., 1970, Scipio Africanus, soldier and politician, Londres, p.29-30. 